\title{
Un acercamiento de la nueva tecnología en los diferentes sectores de la sociedad
}

\section{An approach of the new technology in the different sectors of society}

\section{Uma abordagem da nova tecnologia nos diferentes setores da sociedade}

\author{
Erika C. Bernal-Barcia ${ }^{\mathrm{I}}$ \\ erica.bernal19@gmail.com \\ Johnny G. Rodriguez Sánchez II \\ johnnymabel@gmail.com
}

Recibido: 02 de mayo de $2017 *$ Corregido: 28 de julio de $2017 *$ Aceptado: 14 de septiembre 2017

\footnotetext{
${ }^{\text {I } I n g e n i e r a ~ e n ~ s i s t e m a s, ~ P r o g r a m a ~ d e ~ R e v a l i d a c i o ́ n ~ d e ~ l a ~ M a e s t r i ́ a ~ d e ~ G e s t i o ́ n ~ E s t r a t e ́ g i c a ~ d e ~ T e c n o l o g i ́ a s ~ d e ~ l a ~}$ Información, Facultad de Ingeniería; Universidad de Cuenca, Campus Central, Cuenca, Ecuador. ${ }^{\text {II }}$ Magister en Gestión Empresarial, Ingeniero Comercial, Docente de la Universidad Laica Eloy Alfaro de Manabí, Manta, Ecuador.
} 
Un acercamiento de la nueva tecnología en los diferentes sectores de la sociedad

\section{Resumen}

Las nuevas tecnologías, entendidas como los dispositivos digitales que se pueden conectar con un ordenador o con internet, son probablemente las herramientas más potentes, versátiles y ubicuas que la sociedad haya conocido. A nivel social las telecomunicaciones, han transformado la sociedad en una sociedad de información. Se ha pasado de una sociedad de la comunicación de masas típica del siglo XX a un desarrollo de la comunicación mediática, de carácter virtual. A tal efecto se realizó una revisión bibliográfica, para exponer algunos aspectos relacionados con la tecnología en las diferentes esferas de la sociedad.

Palabras clave: tecnología; sector de la sociedad.

\section{Abstract}

New technologies, understood as digital devices that can be connected to a computer or the Internet, are probably the most powerful, versatile and ubiquitous tools that society has ever known. At the social level, telecommunications have transformed society into an information society. It has moved from a society of mass communication typical of the twentieth century to a development of media communication, virtual character. To this end, a bibliographic review was carried out to present some aspects related to technology in the different spheres of society.

Keywords: technology; sector of society.

\section{Resumo}

As novas tecnologias, entendidas como dispositivos digitais que podem ser conectados a um computador ou à Internet, são provavelmente as ferramentas mais poderosas, versáteis e onipresentes que a sociedade já conheceu. No plano social, as telecomunicações transformaram a sociedade em uma sociedade da informação. Mudou-se de uma sociedade de comunicação de massa típica do século XX para um desenvolvimento de comunicação de mídia, caráter virtual. Para tanto, foi realizada uma revisão bibliográfica para apresentar alguns aspectos relacionados à tecnologia nas diferentes esferas da sociedade.

Palavras chave: tecnologia; setor da sociedade.

\section{Introducción}

4 Vol. 3, núm 4, octubre, 2017, pp. 3-12

Erika C. Bernal Barcia, Johnny G. Rodríguez Sánchez 
Un acercamiento de la nueva tecnología en los diferentes sectores de la sociedad

Fueron necesarios treinta y ocho años, para que la radio reuniera cincuenta millones de usuarios. La televisión tardó trece años en conseguir el mismo público. La internet lo logró en tan sólo cuatro años (Annan, 2000) y ha permitido que los conceptos de comunicación e información se desarrollen en niveles que ninguna otra infraestructura tecnológica ha obtenido. Su expansión fue algo sin precedentes en la historia; no surgió con normas determinadas más allá de los protocolos básicos de comunicación, ya que no se podían prever los problemas que podrían aparecer. (Buxarrais Estrada, M R y Ovide, E. 2011).

Hoy en día los progresos de la denominada tecnología de la información, que abarcan los equipos y aplicaciones informáticas y las telecomunicaciones, están teniendo un gran efecto.

Sin lugar a dudas, las nuevas tecnologías han llevado consigo un cambio espectacular y drástico en toda la esfera de la sociedad. En los últimos años cabe destacar a internet como elemento revolucionario, seguido de la telefonía móvil. En escaso tiempo el internet se ha hecho imprescindible en cualquier sector de la sociedad.

La mayoría de la sociedad realiza un uso diario del ordenador, cuya utilización tiene lugar sobre todo en el hogar y en el centro de trabajo. Por tanto podemos decir que tiene como objetivo laboral.

El vertiginoso desarrollo de la ciencia y la técnica, ha irrumpido en todos los ámbitos y niveles sociales, de modo que en los tiempos actuales la nueva tecnología se ha convertido en uno de los productos fundamentales del consumo de la modernidad.

\section{Desarrollo}

\section{La cultura digital y los movimientos sociales}

Internet ha adquirido gran importancia como instrumento para concebir nuevas manifestaciones, organizaciones y movimientos sociales alrededor de causas en donde la regulación es dada por los individuos implicados. La red como catalizadora de cambios y revoluciones se pone sobre la mesa frente a manifestaciones que han usado Internet como instrumentos de reacción contra regímenes políticos y sociales. (Sojo William. 2015).

5 Vol. 3, núm 4, octubre, 2017, pp. 3-12

Erika C. Bernal Barcia, Johnny G. Rodríguez Sánchez 
Un acercamiento de la nueva tecnología en los diferentes sectores de la sociedad

En continuidad con la influencia de Internet en la sociedad, están los nuevos lenguajes y las nuevas formas de realizar algunas actividades, tales como enviar mensajes de texto, el envío de mensajes de 140 caracteres, la comunicación por e-mail o a través de diversas aplicaciones, han modificado comportamientos y por ende la manera de pensar y hasta de razonar del individuo en la postmodernidad.

El teléfono celular, las tablets, y otros dispositivos, se han convertido en algo más que un accesorio, de tal manera que resulta extraño que alguien en una reunión, no posea un dispositivo de comunicación inteligente. (Sojo William. 2015).

\section{Importancia de las TIC para la gestión empresarial}

Las Tecnologías de la Información y las Comunicaciones (TIC), son cada vez más usadas para el apoyo y automatización de todas las actividades de las empresas. Gracias a ellas, las organizaciones han conseguido obtener importantes beneficios, entre los que caben mencionar la mejora de sus operaciones, llegada a una mayor cantidad de clientes, la optimización de sus recursos, la apertura a nuevos mercados, un conocimiento más profundo acerca de las necesidades de la clientela para brindarles un servicio de mejor calidad y una comunicación más fluida, no sólo con sus empleados sino también con sus clientes y proveedores.

En pocas palabras, las TIC les permiten lograr aumentar considerablemente su eficiencia. (Importancia de las TIC para la gestión empresarial. 2013).

Con certeza, la utilización de las TIC en las diferentes áreas de las compañías ha propiciado un ahorro de costos y tiempo, ayudándoles a su vez con una mejor gestión de los flujos de información. (Importancia de las TIC para la gestión empresarial. 2013).

Vol. 3, núm 4, octubre, 2017, pp. 3-12
Erika C. Bernal Barcia, Johnny G. Rodríguez Sánchez 
Un acercamiento de la nueva tecnología en los diferentes sectores de la sociedad

\section{Sus ventajas en la organización}

Las TICs son esenciales para mejorar la productividad de las empresas, la calidad, el control y facilitar la comunicación, entre otros beneficios, aunque su aplicación debe llevarse a cabo de forma inteligente. (De Jesús Reyes J. 2013).

El mero hecho de introducir tecnología en los procesos empresariales, no es garantía de gozar de estas ventajas. Para que la implantación de nueva tecnología produzca efectos positivos hay que cumplir varios requisitos: tener un conocimiento profundo de los procesos de la empresa, planificar detalladamente las necesidades de tecnología de la información e incorporar los sistemas tecnológicos paulatinamente, empezando por los más básicos. (De Jesús Reyes J. 2013).

Antes de añadir un componente tecnológico, hay que conocer bien la organización y/o empresa. Se ha descubierto que el $90 \%$ de las veces, el fracaso no es debido al software ni a los sistemas, sino al hecho de que la gente no tiene suficientes conocimientos sobre su propia empresa o sus procesos empresariales. (De Jesús Reyes J. 2013).

Las Tecnologías de la Información y la Comunicación han transformado la manera de trabajar y gestionar recursos. Las TIC son un elemento clave para hacer que el trabajo sea más productivo: agilizando las comunicaciones, sustentando el trabajo en equipo, gestionando las existencias, realizando análisis financieros, y promocionando nuestros productos en el mercado. (De Jesús Reyes J. 2013).

El buen uso de las TIC permite a las empresas producir más cantidad, más rápido, de mejor calidad, y en menos tiempo y le ayudan a la competitividad. (De Jesús Reyes J. 2013).

\section{Contexto actual de las prácticas educativas}

Las Tecnologías de la Información y la Comunicación (TICs), han atravesado todos los aspectos de nuestra vida, cambiando nuestra visión del mundo. En consecuencia, también se han modificado y complejizado los patrones de acceso al conocimiento y de relación interpersonal. (Zangara A. 2014).

7 Vol. 3, núm 4, octubre, 2017, pp. 3-12

Erika C. Bernal Barcia, Johnny G. Rodríguez Sánchez 
Un acercamiento de la nueva tecnología en los diferentes sectores de la sociedad

Se ha escrito muchísimo sobre el tema de los cambios tecnológicos, las diferentes miradas sobre la realidad y las consecuencias que estos cambios producen y producirán en el desarrollo de las ciencias y en el fortalecimiento del trabajo interdisciplinario y multidisciplinario. Podemos ver que el mundo se está transformando rápidamente, y con él todas las actividades humanas. La rapidez con que se producen algunos de estos cambios que se dan a todo nivel, tanto en la esfera científica como tecnológica, geográfica, política y hasta moral, nos impacta y nos obliga a hacer importantes y permanentes esfuerzos de adaptación. (Zangara A. 2014).

Este mundo tecnológico, cada vez más complejo, nos desafía a volver, una vez más, sobre las ideas del aprender y del enseñar.

En la actualidad los sistemas educativos de todo el mundo se enfrentan al desafío de utilizar las Tecnologías de la Información y la Comunicación para proveer a sus alumnos con las herramientas y conocimientos necesarios que se requieren en el siglo XXI.

Al respecto, la UNESCO (2004) señala que en el área educativa los objetivos estratégicos apuntan a mejorar la calidad de la educación, por medio de la diversificación de contenidos y métodos, la promoción de la experimentación, la innovación, la difusión y el uso compartido de información y de buenas prácticas, la formación de comunidades de aprendizaje y estimulación de un diálogo fluido sobre las políticas a seguir. Con la llegada de las tecnologías, el énfasis de la profesión docente está cambiando desde un enfoque centrado en el profesor que se basa en prácticas alrededor del pizarrón y el discurso, basado en clases magistrales, hacia una formación centrada principalmente en el alumno dentro de un entorno interactivo de aprendizaje.

En el marco de las observaciones anteriores, las TICs son la innovación educativa del momento y permiten a los docentes y alumnos cambios determinantes en el quehacer diario del aula y en el proceso de enseñanza-aprendizaje de los mismos, brindan herramientas que favorecen a las escuelas que no cuentan con una biblioteca ni con material didáctico. Estas tecnologías permiten entrar a un mundo nuevo lleno de información de fácil acceso para los docentes y alumnos. De igual manera, facilitan el ambiente de aprendizaje, que se adaptan a nuevas estrategias que permiten el desarrollo

8 Vol. 3, núm 4, octubre, 2017, pp. 3-12

Erika C. Bernal Barcia, Johnny G. Rodríguez Sánchez 
Un acercamiento de la nueva tecnología en los diferentes sectores de la sociedad

cognitivo creativo y divertido en las áreas tradicionales del currículo. (Importancia de las TIC en la educación básica regular. 2014).

Con el uso de las computadoras o TICs, los estudiantes desarrollan la capacidad de entendimiento, de la lógica, favoreciendo así el proceso del aprendizaje significativo en los alumnos. (Importancia de las TIC en la educación básica regular. 2014).

Cabe resaltar la importancia de las TICs en las escuelas, por el nivel cognitivo que mejorará en los niños y los docentes, al adquirir un nuevo rol y conocimientos, como conocer la red y cómo utilizarla en el aula e interactuar entre todos con los beneficios y desventajas. (Importancia de las TIC en la educación básica regular. 2014).

Es evidente entonces, la incorporación de las TICs en la educación tiene como función ser un medio de comunicación, canal de comunicación e intercambio de conocimiento y experiencias. Son instrumentos para procesar la información y para la gestión administrativa, fuente de recursos, medio lúdico y desarrollo cognitivo. Todo esto conlleva a una nueva forma de elaborar una unidad didáctica $y$, por ende, de evaluar debido a que las formas de enseñanza y aprendizaje cambian, el profesor ya no es el gestor del conocimiento, sino que un guía que permite orientar al alumno frente su aprendizaje: En este aspecto, el alumno es el "protagonista de la clase", debido a que es él quien debe ser autónomo y trabajar en colaboración con sus pares.( Importancia de las TIC en la educación básica regular. 2014).

Según Fernández Prieto (2014), la presencia del ordenador en todos los ámbitos de nuestra sociedad hace inevitable su uso en entornos educativos y, por tanto, exige una profunda reflexión para descubrir sus mejores potencialidades educativas y su adaptación a la actividad educativa cotidiana. La implantación de las Nuevas Tecnologías en el mundo educativo, abre un horizonte de posibilidades y recursos que pueden y deben enriquecer los procesos de enseñanza y de aprendizaje.

La implantación de las nuevas tecnologías en el mundo educativo abre un horizonte de posibilidades y recursos que pueden y deben enriquecer los procesos de enseñanza y de aprendizaje.

9 Vol. 3, núm 4, octubre, 2017, pp. 3-12

Erika C. Bernal Barcia, Johnny G. Rodríguez Sánchez 
Un acercamiento de la nueva tecnología en los diferentes sectores de la sociedad

\section{Cultura y tecnología}

La tecnología, como parte del ambiente humano, está siempre ligada a la cultura. Esta no solo incluye métodos de sobrevivencia y de producción, sino también la creación del lenguaje, de los sonidos, del arte, etc. Su naturaleza es ambivalente, pues acelera la transferencia de información y de conocimiento.

Según Navarro B. (2014), la cultura es nuestro principal activo y la combinación cultura-internet, nos permite que la misma se pueda compartir con un público más amplio y poder llegar más allá de nuestras fronteras. Pero los ciudadanos no solo quieren tener acceso, también quieren enriquecer su experiencia, interactuar y eso implica algo más. La tecnología permite una nueva forma de relacionarse con el arte, con la cultura, de aprender acentuando las capacidades comunicativas del mundo físico tradicional y aportando un valor incalculable a las obras.

Facilita asimismo la preservación de nuestro patrimonio, generando un gran impacto en la forma en que el conocimiento se construye e intercambia. Tecnología al servicio del ciudadano y de la sociedad en general y para ello el contenido cultural debe formar parte de ese proceso para que nuestra memoria colectiva siga en constante evolución. Cultura es sinónimo de historia. Nos permite entender nuestro pasado, comprender mejor nuestro presente y mirar con decisión hacia el futuro, por tanto conservarla, difundirla y vivirla es clave para nuestra sociedad. (Navarro B. 2014).

\section{La tecnología y la familia}

Los medios de comunicación de hace unos 100 años, influían muy poco en la manera de vivir, de convivir y de educar en las familias. Las familias iban al encuentro de información, tenían más tiempo con los hijos, la madre estaba en casa y había más oportunidad de observar, de influir en la trasmisión de valores, tradiciones, y educar. (Aparicio Moreno M., Zermeño Cataño E. 2010).

Hoy por hoy, en las ciudades los ritmos de vida son muy rápidos, muchas madres trabajan, los hijos pueden llegar a estar solos por muchas horas, hay poco tiempo para educar y las nuevas tecnologías han invadido todo. (Aparicio Moreno M., Zermeño Cataño E. 2010).

10 Vol. 3, núm 4, octubre, 2017, pp. 3-12

Erika C. Bernal Barcia, Johnny G. Rodríguez Sánchez 
Un acercamiento de la nueva tecnología en los diferentes sectores de la sociedad

Cabe agregar que la tecnología influye en la manera de pensar, de convivir, de relacionarse y de actuar de las familias. Las nuevas tecnologías, son medios para comunicarse, relacionarse y expresarse como persona y como cualquier otro medio, pueden utilizarse bien o mal. (Aparicio Moreno M., Zermeño Cataño E. 2010).

En los hogares donde las relaciones son buenas, internet y el celular refuerzan los vínculos; de lo contrario, favorecen el individualismo.

A manera de colofón la tecnología puede resolver muchos problemas de la era moderna, pero hay otros como la guerra, el racismo, el hambre y la violencia que no se pueden solucionar con tecnología. Cuando se implementan técnicas para aliviar problemas, usando la tecnología, muchas veces se ignora la cultura, la política, la economía, las relaciones y hasta el mismo ser humano.

\section{Referencia bibliográfica}

Aparicio Moreno M, Zermeño Cataño E. 2010. Relaciones familiares y nuevas tecnología en el siglo XXI

Annan, K. 2000. We, The Peoples. The Role of the United Nations in the 21st Century. Naciones Unidad: Nueva York, [consulta 25 mayo 2017]. Disponible en: http://www.un.org/millennium/sg/report/full.htm

Buxarrais Estrada, M R, Ovide, E. 2011. El impacto de las nuevas tecnologías en la educación en valores del siglo XXI n. 37, pp.1-14. Disponible en: $<\mathrm{http}: / /$ www.scielo.org.mx/scielo.php?script=sci_arttext\&pid=S1665109X2011000200002\&lng=es\&nrm=iso>

De Jesús reyes J. 2013. Las TICs y la Gestión empresarial. Disponible en: http://www.eoi.es/blogs/mtelcon/2013/02/06/las-tics-y-la-gestion-empresarial

Fernández Prieto M S. 2014 Las nuevas tecnologías en la educación, [consulta 25 mayo 2017]. Disponible en: https://www.uam.es/manuel.fernandez/libro.pdf

11 Vol. 3, núm 4, octubre, 2017, pp. 3-12

Erika C. Bernal Barcia, Johnny G. Rodríguez Sánchez 
Un acercamiento de la nueva tecnología en los diferentes sectores de la sociedad

Importancia de las TIC para la gestión empresarial [sitio web]. 2013. [consulta 25 mayo 2016]. Disponible en: http://www.aniel.es/importancia-de-las-tic-para-la-gestion-empresarial/

Importancia de las TIC en la educación básica regular [sitio web]. 2014 [consulta 25 mayo 2017]. Disponible en: https://educrea.cl/importancia-de-las-tic-en-la-educacion-basica-regular/

NAVARRO B (2014). Cultura y tecnología, [consulta 25 mayo 2017]. Disponible en:http://www.elmundo.es/blogs/elmundo/voces/2014/04/08/cultura-y-tecnologia.html

SOJO WILLIAM. 2015. Influencia e importancia de las TIC en las organizaciones, [consulta 25 mayo 2017]. Disponible en:https://www.gestiopolis.com/influencia-e-importancia-de-las-tic-en-lasorganizaciones/

ZANGARA A. 2014 Uso de nuevas tecnologías en la educación: una oportunidad para fortalecer la práctica docente, [consulta 25 mayo 2017]. Disponible en: http://www.puertasabiertas.fahce.unlp.edu.ar/numeros/n5/zangara|

12 Vol. 3, núm 4, octubre, 2017, pp. 3-12

Erika C. Bernal Barcia, Johnny G. Rodríguez Sánchez 